\title{
ROBUST AERIAL OBJECT TRACKING IN HIGH DYNAMIC FLIGHT MANEUVERS
}

\author{
A. Nussberger ${ }^{\mathrm{a}}$, H. Grabner ${ }^{\mathrm{a}}$, L. Van Gool ${ }^{\mathrm{a}}$ \\ ${ }^{a}$ Computer Vision Laboratory, ETH Zurich, Switzerland - (nussberger, grabner, vangool)@ vision.ee.ethz.ch
}

KEY WORDS: Aircraft, Detect and Avoid, Dynamic Flight, Object Tracking, Optical Sensors, Remotely Piloted Aircraft System

\begin{abstract}
:
Integrating drones into the civil airspace is one of the biggest challenges for civil aviation, responsible authorities and involved companies around the world in the upcoming years. For a full integration into non-segregated airspace such a system has to provide the capability to automatically detect and avoid other airspace users. Electro-optical cameras have proven to be an adequate sensor to detect all types of aerial objects, especially for smaller ones such as gliders or paragliders. Robust detection and tracking of approaching traffic on a potential collision course is the key component for a successful avoidance maneuver. In this paper we focus on the aerial object tracking during dynamic flight maneuvers of the own-ship where accurate attitude information corresponding to the camera images is essential. Because the 'detect and avoid' functionality typically extends existing autopilot systems the received attitude measurements have unknown delays and dynamics. We present an efficient method to calculate the angular rates from a multi camera rig which we fuse with the delayed attitude measurements. This allows for estimating accurate absolute attitude angles for every camera frame. The proposed method is further integrated into an aerial object tracking framework. A detailed evaluation of the pipeline on real collision encounter scenarios shows that the multi camera rig based attitude estimation enables the correct tracking of approaching traffic during dynamic flight, at which the tracking framework previously failed.
\end{abstract}

\section{INTRODUCTION}

Within the upcoming decade aviation will be faced with one of their biggest challenges: the safe integration of Remotely Piloted Aircraft Systems (RPAS) into the civil airspace. The particular roadmaps published by the U.S. and Europe in 2013 [Federal Aviation Administration, 2013b, European RPAS Steering Group, 2013] plan a step by step integration depending on the airspace class and required technologies. The goal is to finally integrate RPAS by 2020-2030 into airspace classes where Visual Flight Rules (VFR) apply. One of the critical enabling technologies to achieve this target is the availability of a technical system that replaces the human 'See and Avoid' from manned aviation. According to recent publications such a 'Detect and Avoid' (DAA) system shall provide an equivalent level of safety compared to a human pilot [International Civil Aviation Organization, 2011, Federal Aviation Administration, 2013a].

A typical Detect and Avoid system has to deal with so called cooperative and non-cooperative traffic. While cooperative traffic usually transmits its own position via transponder based technologies (e.g. $A D S-B^{1}$ ), non-cooperative traffic has to be detected in a different way. Most of the current research activities with focus on non-cooperative traffic detection and tracking either use a RADAR [Wolfe, 2003, Korn and Edinger, 2008, Owen et al., 2014], electro optical (EO) sensors [Utt et al., 2004, Carnie et al., 2006, Dey et al., 2011, Mejias et al., 2012, Nussberger et al., 2014] or a combination of them [Forlenza et al., 2012]. While RADAR allows to measure the distance to a given object it might fail to detect small airspace users such as gliders or paragliders. EO sensors on the other hand consume much less power and space but estimating a distance to the detected object is nontrivial. Both technologies have in common that the detection of traffic in front of terrain is a very challenging task usually resulting in a large number of false tracks. Therefore most of the published results are limited to detect and track aerial objects in

${ }^{1}$ ADS-B is a cooperative, standardized technology to transmit the own position and velocity at a fixed interval. The maximum range is given by the aircraft transponder power and can exceed $100 \mathrm{~km}$.

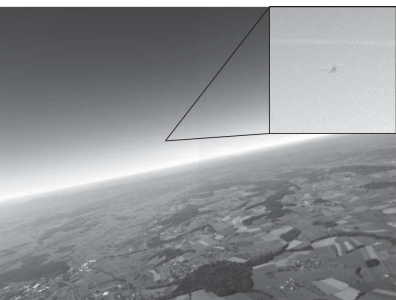

(a) $\mathrm{t}=0 \mathrm{~s} / \mathrm{d}=1225 \mathrm{~m}$

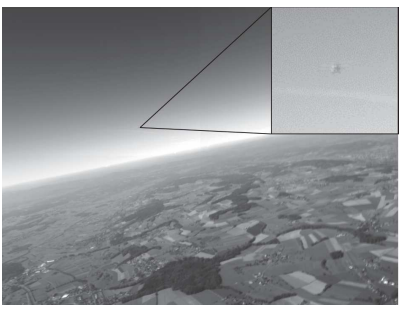

(c) $\mathrm{t}=2 \mathrm{~s} / \mathrm{d}=995 \mathrm{~m}$

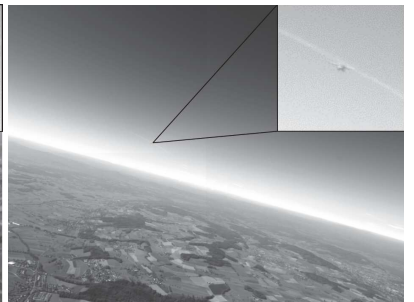

(b) $\mathrm{t}=1 \mathrm{~s} / \mathrm{d}=1110 \mathrm{~m}$

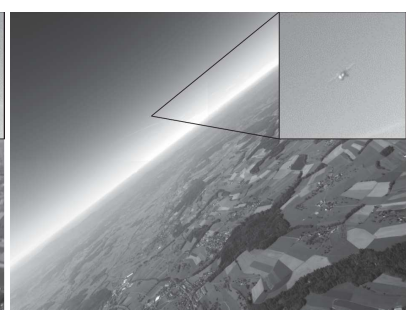

(d) $t=3 \mathrm{~s} / \mathrm{d}=880 \mathrm{~m}$
Figure 1: Example images of an approaching traffic aircraft recorded from an aerial platform during a dynamic flight maneuver. The upper right corner shows a magnified cutout of the incoming traffic. Notice that sky as background eases detection.

the sky part of the sensor, a strong limitation when operating at low altitude or in a mountainous area.

In [Nussberger et al., 2014] we proposed an aerial object tracking framework based on EO sensors which is able to detect and track aerial objects in the sky region of an image as well as in front of terrain. With a multi-layer filter pipeline false tracks from ground clutter and distractor objects such as distant clouds or dirt on the lens are suppressed. At the core of the tracking framework a unit sphere is used, centered at the position of the own-ship and independent of its attitude. All detections from the multi camera rig shown in Figure 2 are projected onto the surface of this sphere where a constant angular velocity model is applied for the tracking. This setup allows decoupling the tracking of aerial objects from the sensor reference frames. Multiple overlapping or 


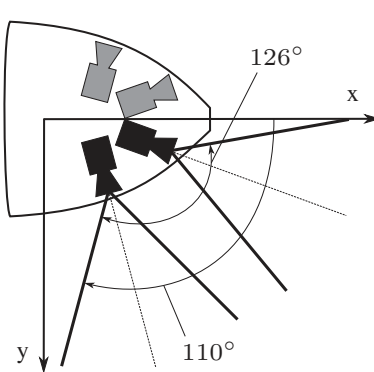

(a) Multi camera rig

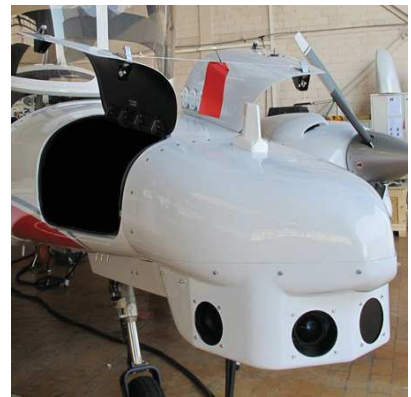

(b) Custom nose-pod
Figure 2: Overview of the multi camera rig in the sensor nosepod. As shown in the left figure, only two of the possible four cameras are mounted in the pod which was sufficient to record the relevant collision scenarios and cover approximately half of the recommended field of view for a Detect and Avoid system [ASTM International, 2007]: $\pm 110^{\circ}$ horizontally and $\pm 15^{\circ}$ vertically.

non-overlapping sensors can easily be integrated by a proper calibration. However, the proposed framework is heavily depending on accurate attitude measurements because otherwise new detections will be projected to inconsistent positions on the unit sphere resulting in multiple tracks or no track at all. While this problem is negligible during level flight and smooth maneuvers, it is a serious issue to consider for large sensor displacements in between consecutive frames. This is the issue dealt with in this paper.

Large sensor displacements usually occur within high dynamic flight maneuvers, e.g. due to a sudden avoidance maneuver. It is obvious that losing track of the aerial object the system is currently avoiding is very unfavorable in terms of situational awareness and conflict resolution. The availability of high precision attitude angles corresponding to the sensor sample rates from the DAA system is a critical issue in RPAS DAA systems. The DAA functionality is typically integrated as an additional sub-system connected to the aircraft autopilot as shown in Figure 3. This system architecture is a result of existing RPAS functionality, safety and certification requirements. In addition it supports the specific interfaces and high processing power required for the DAA sensors - especially when using optical cameras or a RADAR. Within this configuration all meta data such as position and attitude information is provided by the aircraft autopilot. Depending on the interface the (third-party) autopilot manufacturer provides, this will introduce unknown delays and dynamics.

A lot of research has been done in the area of visual odometry (VO) [Nistér et al., 2004, Kneip et al., 2011, Forster et al., 2014] or simultaneous localization and mapping (SLAM) [Davison, 2003, Klein and Murray, 2007, Engel et al., 2014] where typically the vehicle rotation is estimated together with its translation. However, these methods either require high frame rate cameras or they are computationally expensive, especially if we are only interested in the rotational component in between consecutive frames.

\begin{tabular}{|c|c|c|c|c|}
\hline $\begin{array}{l}\text { Redundant } \\
\text { Navigation } \\
\text { Sensors }\end{array}$ & $\begin{array}{c}\text { Aircraft } \\
\text { Autopilot }\end{array}$ & $\leftrightarrow$ & $\begin{array}{c}\text { Detect \& Avoid } \\
\text { Sub-System }\end{array}$ & $\begin{array}{c}\text { Detect \& Avoid } \\
\text { Sensors }\end{array}$ \\
\hline
\end{tabular}

Figure 3: Typical Detect and Avoid System Architecture. The aircraft autopilot is directly connected to a number of navigation sensors which are fused to a consistent navigation solution. The Detect and Avoid sub-system receives its meta data such as position and attitude data from the autopilot which introduces unknown delays and dynamics.
In this paper we present an efficient approach to extract the aircraft angular rates from a multi camera rig. By fusing these angular rates with delayed, absolute attitude angles received from the RPAS autopilot we estimate the inter-frame delta angles while keeping the global attitude drift minimal. The proposed attitude estimation method is further integrated into the aerial object tracking framework where we will show improved results based on the same dataset as used in [Nussberger et al., 2015] and additional challenging high dynamic maneuver scenarios. Within these additional scenarios the pilot was manually flying a so called wingrock where the aircraft continuously banks from the left to the right and reverse to induce large ego-motion.

The paper is structured as follows. Section 2. introduces the angular rate estimation based on the multi camera rig and describes the proposed filter to fuse the angular rates with delayed absolute attitude measurements. In Section 3. we show a detailed evaluation of the proposed attitude estimation filter. Section 4. shows the attitude estimation applied to aerial object tracking and in Section 5. we conclude the paper.

\section{ATTITUDE ESTIMATION}

Accurate attitude angles are a key component to efficient and robust tracking in aerial images. In this Section we introduce a method to efficiently calculate the aircraft angular rates from a multi camera rig. In addition we show how to fuse these relative angular rates with delayed, absolute attitude measurements.

\subsection{Requirements for a Pure Rotation Transformation}

One of the main differences between the VO and SLAM research compared to our work is that they usually focus on micro aerial vehicles (MAVs) operating close to the ground. In contrast, the presented experimental DAA system is based on a general aviation (GA) sized RPAS typically operating at altitudes way beyond $500 \mathrm{~m}$ above ground. This is exactly the key property we will use for the efficient calculation of the angular rates from the multi camera rig. With a minimum altitude of $500 \mathrm{~m}$ above ground we can assume a pure rotation in between consecutive frames under the conditions explained below, i.e. conditions under which the own-ships forward translation has no effect on the image.

For a simplified analysis we assume an aircraft as shown in Figure 4 at constant altitude and speed with a forward looking camera with given vertical angular resolution

$$
\theta_{v}=\frac{\text { Vertical field of view }}{\text { Number of vertical pixels }} .
$$

At time instant $t_{1}$ the camera observes a stationary feature on the ground $F^{*}$ under an angle $\alpha$. In the next frame at $t_{2}$ the same feature will be visible under an angle $\alpha^{\prime}$. For the pure rotation case we have to make sure the feature is projected to the same pixel for both time instances. This is valid as long as the angular change of the feature in between the two frames is smaller than the vertical camera resolution:

$$
\alpha^{\prime}-\alpha \leq \theta_{v} .
$$

With the horizontal distance $d_{x}$ of the feature $F^{*}$ to the own-ship at time $t_{1}$, the aircraft speed $v_{a c f t}$ and $d_{f}=v_{a c f t} \cdot\left(t_{2}-t_{1}\right)$ we can express the angles $\alpha$ and $\alpha^{\prime}$ as follows:

$$
\begin{aligned}
\alpha & =\arctan \left(\frac{h}{d_{x}}\right), \\
\alpha^{\prime} & =\arctan \left(\frac{h}{d_{x}-d_{f}}\right) .
\end{aligned}
$$




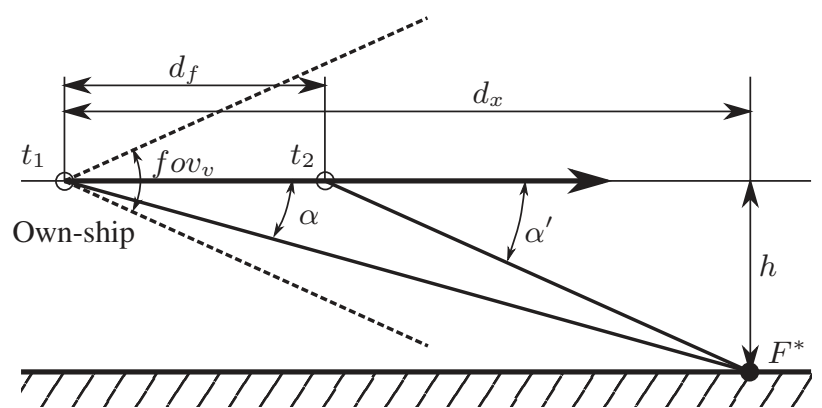

Figure 4: Sketch of an aircraft flying at constant altitude and speed parallel to the ground. The camera with vertical field of view fov $_{v}$ observes a stationary feature $F^{*}$ at two consecutive frames at time instances $t_{1}$ and $t_{2}$ under the corresponding angles $\alpha$ and $\alpha^{\prime}$. If the angular change in between these two angles is smaller than the camera angular resolution $\theta_{v}$ we have a pure rotation in between the two consecutive frames.

After combining these equations we can express the minimum required altitude for the pure rotation assumption to hold, given the aircraft speed $v_{a c f t}$, the camera vertical angular resolution $\theta_{v}$ and a given 'feature angle' $\alpha$ :

$$
h_{\text {min }} \geq \frac{\tan \left(\alpha+\theta_{v}\right) \cdot \tan (\alpha) \cdot v_{a c f t} \cdot\left(t_{2}-t_{1}\right)}{\tan \left(\alpha+\theta_{v}\right)-\tan (\alpha)} .
$$

In Table 1 we show different examples of parameters with the corresponding resulting minimum altitude for common aircraft speeds of 100 and 150 knots. In addition to the original vertical image size of 2472 pixels we also provide results for downsampled images. They show that the assumption of a pure rotation in between two consecutive frames is satisfied as soon as we use a properly down-sampled image and the aircraft operates above approximately $500 \mathrm{~m}$.

\begin{tabular}{|c|c|c|c|c|}
\hline \multicolumn{4}{|c|}{ Parameters } & \multirow{2}{*}{$\begin{array}{c}\text { Result } \\
h_{\min } \\
{[\text { meter }]}\end{array}$} \\
\hline $\begin{array}{c}v_{\text {acft }} \\
{[k n o t s]}\end{array}$ & $\begin{array}{l}\text { px } x_{\text {ver }} \\
{[\text { pixel }]}\end{array}$ & $\begin{array}{c}\theta_{v} \\
{[\operatorname{deg} / p x]}\end{array}$ & $\begin{array}{c}\alpha \\
{[\operatorname{deg}]}\end{array}$ & \\
\hline 100 & 2472 & 0.02 & 10 & 214 \\
\hline 100 & 2472 & 0.02 & 20 & 829 \\
\hline 100 & 2472 & 0.02 & 30 & 1772 \\
\hline 100 & 2472 & 0.02 & 40 & 2927 \\
\hline 100 & 618 & 0.08 & 10 & 54 \\
\hline 100 & 618 & 0.08 & 20 & 208 \\
\hline 100 & 618 & 0.08 & 30 & 444 \\
\hline 100 & 618 & 0.08 & 40 & 733 \\
\hline 150 & 618 & 0.08 & 10 & 81 \\
\hline 150 & 618 & 0.08 & 20 & 314 \\
\hline 150 & 618 & 0.08 & 30 & 670 \\
\hline 150 & 618 & 0.08 & 40 & 1106 \\
\hline
\end{tabular}

Table 1: Comparison of different parameter configurations required for the pure rotation assumption in between two consecutive frames and the resulting minimal required altitude $h_{m i n}$ : aircraft speed $v_{a c f t}$, vertical resolution in pixel $p x_{v e r}$, vertical angular resolution $\theta_{v}$ and feature angle $\alpha$. In addition to the values specified in the table we assume a constant frame rate of $20 \mathrm{fps}$ and a vertical field of view of $51^{\circ}$.

\subsection{Multi Camera Rig Angular Rate Estimation}

The main problem with the current tracking framework is its requirement for accurate attitude measurements which have to correspond precisely to the actual camera motion. Therefore we will directly use the camera as a sensor itself. Because the experimental DAA system is based on multiple cameras we are even able to integrate the motion information from all of them. For the angular rate estimation from the multi camera rig we use the same idea of a unit sphere centered at the current aircraft position as proposed for the tracking. In contrast to the tracking framework the unit sphere is defined with respect to the local aircraft body reference frame (see Figure 5) instead of the local navigation reference frame. Note that for the following steps we assume the multi camera rig to be fully calibrated. Based on the results from the previous subsection we implemented the angular rate estimation as follows:

1. For every camera we down-sample the current frame to reduce the overall computational cost. As shown in Table 1 the resulting angular resolution is still below $0.1^{\circ}$ even if the image is down-sampled by a factor of four.

2. In the next step, SURF features are extracted. Most of them obviously from the heavily textured terrain part of the images. Because of the available multi camera setup the terrain can be expected to be visible in at least two of the four cameras during all common flight maneuvers.

3. The detected features are now projected onto the surface of the unit sphere using the corresponding extrinsic camera calibration as shown in Figure 5. These features are then matched in between the unit sphere of the current time step and the previous one, which results in a set of point correspondences related by a rotation matrix $\boldsymbol{R}_{3 \times 3}$.

4. Based on this set of point correspondences we calculate $\boldsymbol{R}_{3 x 3}$ according to [Challis, 1995] using a RANSAC based approach to account for possible outliers. The rotation matrix we get yields the delta angles from the previous to the current frame. From the rotation matrix we extract the Euler angles. By dividing them with the sample time we finally get the angular rates $\boldsymbol{\omega}_{c a m}=[p, q, r]^{\top}$.

A brief overview of the complete angular rate estimation algorithm in pseudo code is shown in Figure 6.

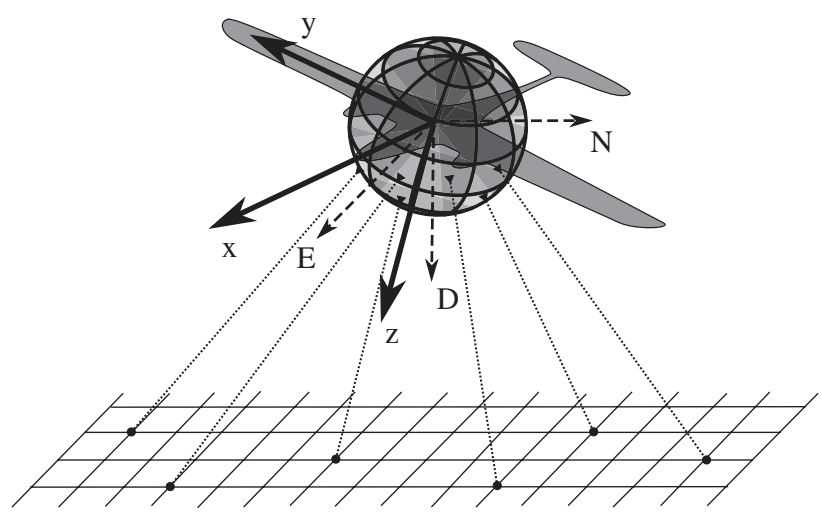

Figure 5: Mapping of features from the ground onto the surface of the unit sphere (dotted lines) centered at the aircraft position in body reference frame (x,y and $\mathrm{z}$-axis). Each camera provides features to the sphere at different locations depending on the visible terrain in the camera field of view. The navigation reference frame is indicated by the dashed North, East and Down (NED) vectors. 


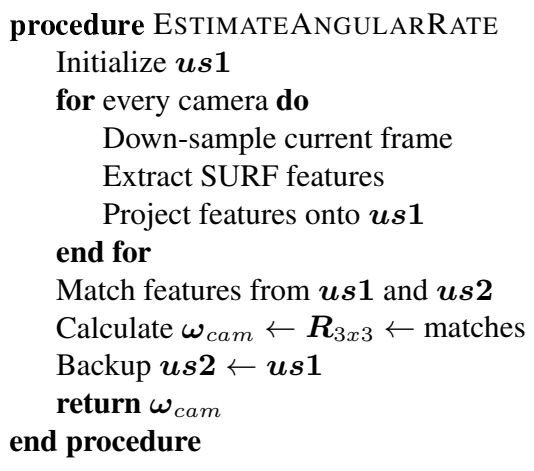

Figure 6: Basic algorithm to estimate the angular rate $\boldsymbol{\omega}_{\text {cam }}$ with the multi camera rig. The features of the current camera frames are projected onto the first unit sphere $u s \mathbf{1}$, whereas the features from the previous time step are available in $\boldsymbol{u s} \mathbf{2}$.

\subsection{Fusion of Camera Angular Rates with Delayed Absolute Attitude Measurements}

With the estimated angular rates from the multi camera rig and the available (but delayed) absolute attitude measurement from the aircraft autopilot we setup an indirect Kalman filter to get a delay compensated absolute attitude estimate. For the attitude representation we use a quaternion based approach. This allows a straight forward integration of angular rates and avoids common issues such as 'gimbal lock' when using Euler Angles. Note that we use the Hamilton notation where the first value of the quaternion corresponds to the scalar component: $\boldsymbol{q}=\left[q_{0}, \boldsymbol{q}_{1-3}\right]^{\top}$.

The system model is based on the integration of the angular rates with the assumption of $\dot{\boldsymbol{\omega}}=0$, driven by Gaussian noise. While this is not strictly correct, it is an acceptable approximation because we directly measure this value by $\boldsymbol{\omega}_{\text {cam }}$. A different approach could be to directly use $\boldsymbol{\omega}_{\text {cam }}$ as input but then we would have to take special care if for once the calculation of $\boldsymbol{\omega}_{\text {cam }}$ fails. The continuous time system state dynamics ar given as

$$
\begin{aligned}
\dot{\boldsymbol{q}} & =\frac{1}{2} \cdot \boldsymbol{Q}(\boldsymbol{\omega}) \cdot \boldsymbol{q} \\
\dot{\boldsymbol{\omega}} & =\boldsymbol{n}_{\omega}
\end{aligned}
$$

where $\boldsymbol{Q}$ is the quaternion matrix function [Diebel, 2006] and $\boldsymbol{n}_{\omega}$ the angular acceleration noise. As already stated before we use an indirect Kalman filter where the errors are part of the filter state and the error dynamics are modeled. The correct attitude is calculated by multiplying the estimated $\hat{\boldsymbol{q}}$ with an error rotation $\delta \boldsymbol{q}$. Therefore the resulting error model is given by

$$
\begin{aligned}
\delta \boldsymbol{q} & =\hat{\boldsymbol{q}}^{-1} \otimes \boldsymbol{q} \\
\Delta \boldsymbol{\omega} & =\boldsymbol{\omega}-\hat{\boldsymbol{\omega}}
\end{aligned}
$$

Because the angular errors are usually small, we are able to apply the small angle approximation for quaternions

$$
\delta \boldsymbol{q} \approx\left[\begin{array}{ll}
1 & \frac{1}{2} \delta \boldsymbol{\theta}
\end{array}\right]^{\top}
$$

which reduces the number of parameters to three and avoids an overdetermined attitude representation within the filter. This results in the error state vector

$$
\boldsymbol{x}_{6 \times 1}=\left[\begin{array}{ll}
\delta \boldsymbol{\theta}_{3 \times 1}^{\top} & \Delta \boldsymbol{\omega}_{3 \times 1}^{\top}
\end{array}\right]^{\top}
$$

with the corresponding continuous time error state dynamics

$$
\begin{aligned}
\delta \dot{\boldsymbol{\theta}} & =-\boldsymbol{\omega}_{\times} \cdot \delta \boldsymbol{\theta} \\
\Delta \dot{\boldsymbol{\omega}} & =\boldsymbol{n}_{\omega}
\end{aligned}
$$

where $\boldsymbol{\omega}_{\times}$is the skew symmetric matrix of $\boldsymbol{\omega}$. In [Trawny and Roumeliotis, 2005], a detailed derivation of Equation (12) and the corresponding discrete formulation is presented.

The prediction step of the filter is calculated according to the following equations where $\boldsymbol{I}$ is the identity matrix, $\hat{\boldsymbol{P}}$ the estimated covariance, $\boldsymbol{F}$ the discretized error system matrix and $\boldsymbol{V}$ the error process noise. For the integration of the quaternion a zero order approximation is used.

$$
\begin{aligned}
& \hat{\boldsymbol{\omega}}_{k+1, k}=\hat{\boldsymbol{\omega}}_{k, k} \\
& \hat{\boldsymbol{q}}_{k+1, k}=\left(\boldsymbol{I}+\Delta t \cdot \frac{1}{2} \cdot \boldsymbol{Q}\left(\boldsymbol{\omega}_{k+1, k}\right)\right) \cdot \boldsymbol{q}_{k, k} \\
& \hat{\boldsymbol{P}}_{k+1, k}=\boldsymbol{F}_{k} \cdot \hat{\boldsymbol{P}}_{k, k} \cdot \boldsymbol{F}_{k}^{\boldsymbol{\top}}+\boldsymbol{V}_{k}
\end{aligned}
$$

Within the filter update step we have to incorporate the delayed attitude measurement. The classical approach would be to augment the filter state [Tasoulis et al., 2007] and setup the measurement matrix $\boldsymbol{H}$ accordingly. In our setup we avoid increasing the filter state by buffering previous states. Instead we just save the history of angular rates to project the delayed attitude measurement to the current time step. Note that we assume a constant delay for the attitude measurements which we determine once from recorded data. If the delay is not constant we could estimate it e.g. by calculating the cross correlation [Kelly and Sukhatme, 2010] in between the angular rates from the camera and the ones from the aircraft autopilot. To calculate the filter update, we proceed as follows:

1. Propagate delayed attitude measurement via quaternion integration to current time step

2. Compute residual $\boldsymbol{\nu}$ for the angular rates and the attitude angles using the small angle approximation

3. Compute Kalman gain with measurement matrix $\boldsymbol{H}$ and measurement noise $\boldsymbol{W}$ :

$$
\begin{aligned}
\boldsymbol{K}_{k+1} & =\hat{\boldsymbol{P}}_{k+1, k} \cdot \boldsymbol{H}^{\boldsymbol{\top}} \cdot \boldsymbol{S}^{-1} \\
\boldsymbol{S} & =\boldsymbol{H} \cdot \hat{\boldsymbol{P}}_{k+1, k} \cdot \boldsymbol{H}^{\boldsymbol{\top}}+\boldsymbol{W}
\end{aligned}
$$

4. Compute correction:

$$
\left[\begin{array}{c}
\Delta \boldsymbol{\omega} \\
\delta \boldsymbol{\theta}
\end{array}\right]_{k+1}=\boldsymbol{K}_{k+1} \cdot \boldsymbol{\nu}_{k+1}
$$

5. Update states and covariance matrix:

$$
\begin{aligned}
\hat{\boldsymbol{\omega}}_{k+1, k+1}= & \hat{\boldsymbol{\omega}}_{k+1, k}+\Delta \boldsymbol{\omega}_{k+1} \\
\hat{\boldsymbol{q}}_{k+1, k+1}= & \hat{\boldsymbol{q}}_{k+1, k} \otimes \delta \hat{\boldsymbol{q}}_{k+1} \\
\hat{\boldsymbol{P}}_{k+1, k+1}= & \left(\boldsymbol{I}-\boldsymbol{K}_{k+1} \cdot \boldsymbol{H}\right) \cdot \hat{\boldsymbol{P}}_{k+1, k} \\
& \cdot\left(\boldsymbol{I}-\boldsymbol{K}_{k+1} \cdot \boldsymbol{H}\right)^{\top} \\
& +\boldsymbol{K}_{k+1} \cdot \boldsymbol{W} \cdot \boldsymbol{K}_{k+1}^{\top}
\end{aligned}
$$

\section{EVALUATING THE ATTITUDE ESTIMATION}

This section focuses on the evaluation of our attitude estimation filter. After showing results for the multi camera rig angular rate estimation we compare the delayed attitude measurements from the autopilot with the output of our filter. 


\subsection{Multi Camera Rig Angular Rate Estimation Analysis}

The angular rate calculation from the multi camera rig is heavily relying on robust features in between consecutive frames. Especially for dynamic flight maneuvers the results improve with a uniform distribution of features on the unit sphere. Therefore the more cameras provide features, the better. As shown in Figure 2, the current multi camera rig configuration has two built-in cameras of the planned four. In Figure 7 we show example plots of the angular rate $\mathrm{p}$ around the roll axis calculated from the camera rig for one of the wingrock scenarios. Results are presented for the front camera only and for both cameras. We can see that especially for the large displacements at around $\pm 0.6 \mathrm{rad} / \mathrm{s}$ the accuracy is increased if the second camera is included. On the other hand the accuracy usually drops if the aircraft banks to the left because then the right camera points to the sky and the two left cameras are not yet available in the camera rig. This can result in errors in the uprising part of the angular rate $\mathrm{p}$ as shown in Figure 7(b). The corresponding number of features over time is given by Figure 7(c), where a significant drop in number of features for the right camera is visible in between 6.5 and 8.5 seconds. This is exactly while the camera is pointing to the blue sky.

\subsection{Attitude Estimation Filter Analysis}

The attitude data from the aircraft autopilot is assumed to have a constant delay. By fusing these delayed attitude measurements with the angular rates from the multi camera rig according to the method presented in Subsection 2.3, we can compensate for the delay as shown in Figure 8. There we show a comparison of the roll angle from the aircraft autopilot and the output from the filter. The roll angle is obviously the most significant angle during a wingrock. Because the delay is rather small compared to the scenario duration, two zoomed cutouts are shown.

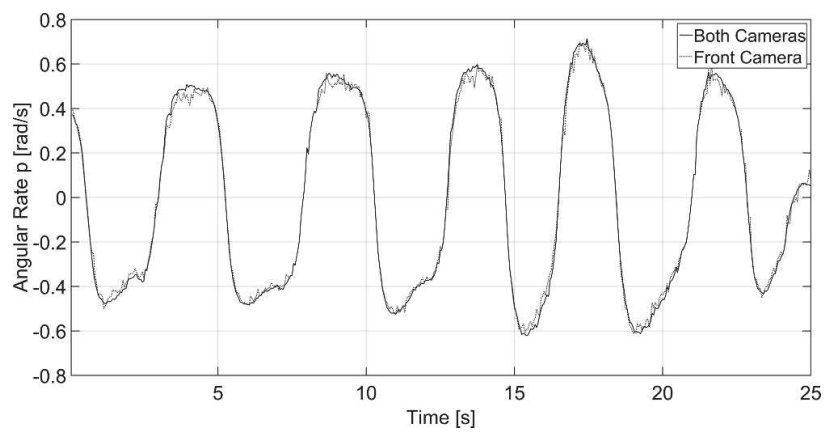

(a) Angular rate $\mathrm{p}$ from the multi camera rig during a wingrock scenario

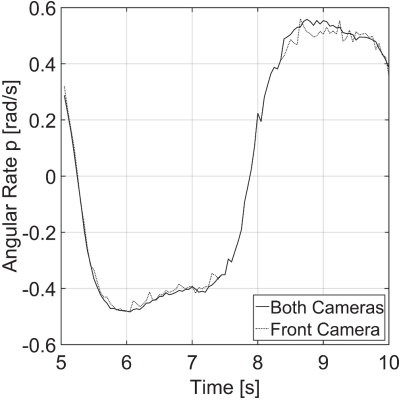

(b) Angular rate p cutout

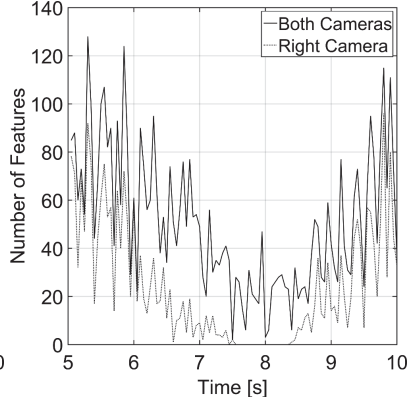

(c) Number of features cutout
Figure 7: Angular rate of the roll axis calculated from the multi camera rig during a wingrock scenario. Results are shown for the front camera only and both cameras. As shown in the bottom right figure, the influence of the right camera drops if the aircraft banks to the left.
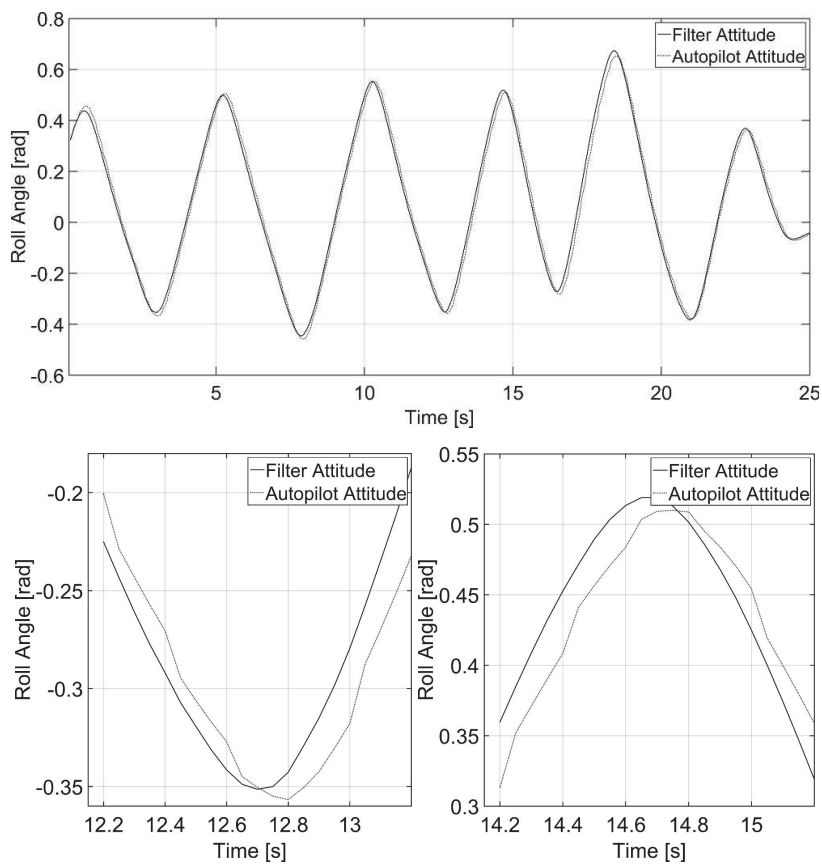

Figure 8: Comparison of the roll angle from the aircraft and the attitude filter during a wingrock scenario with two cutouts at 12.7 and 14.7 seconds respectively.

\section{ATTITUDE ESTIMATION APPLIED TO AERIAL OBJECT TRACKING}

The motivation for calculating the angular rates from the multi camera rig and fusing them with the delayed attitude measurements from the RPAS autopilot is to get accurate absolute attitude angles corresponding to the current camera frame. This enables improved tracking performance during highly dynamic maneuvers, e.g. during an automatic avoidance. In this section we give a brief overview of the extended aerial object tracking framework and show a detailed evaluation based on existing and new scenarios.

\subsection{Extended Aerial Object Tracking Framework}

The aerial object tracking framework shown in Figure 9 has as main steps object detection, detection fusion and tracking. Additional meta information such as the GPS position and the attitude measurements from the aircraft autopilot or a digital terrain model (DTM) are available throughout the pipeline. A small delay in the GPS position can be neglected in the given setup, because even at 150 knots the aircraft travels less than $4 \mathrm{~m}$ in between two frames. The delayed attitude measurements are much more critical, especially within the detection fusion step where the detections from all sensors and detectors are projected onto the tracking sphere. Therefore we extend our existing aerial object tracking framework from [Nussberger et al., 2015] with an additional 'Attitude Estimation' block as preprocessing step based on the results presented before. For the 'Horizon Estimation' and 'Lens Flare Detection' blocks accurate attitude angles as inputs are not that critical because both can deal with angular errors of a few degrees. There having accurate attitude angles reduces computational costs within the iterative refinement. Theoretically also the tracking parameters could be adjusted to deal with inaccurate attitude measurements but this would significantly degrade the performance by creating a large number of false tracks. With the availability of precise attitude angles the tracker parameters can be tuned such that the probability of assigning detections to a non-corresponding track is significantly reduced. 


\begin{tabular}{|c||c|c||c|c|c||c|c|c|}
\hline \multicolumn{1}{||}{} & \multicolumn{2}{c||}{ Scenarios } & \multicolumn{3}{c||}{ Results without } & \multicolumn{3}{c|}{ Results with } \\
Attitude Estimation Filter & \multicolumn{2}{c|}{ Attitude Estimation Filter } \\
& Type & Background & Distance & TTC & $\begin{array}{c}\text { False } \\
\text { Tracks }\end{array}$ & Distance & TTC & $\begin{array}{c}\text { False } \\
\text { Tracks }\end{array}$ \\
\hline \hline N & Wingrock & Sky & fails & - & - & $1932 \mathrm{~m}$ & $16.0 \mathrm{~s}$ & 0 \\
\hline O & Wingrock & Terrain & fails & - & - & $1730 \mathrm{~m}$ & $15.3 \mathrm{~s}$ & 0 \\
\hline P & Wingrock & Sky & fails & - & - & $1908 \mathrm{~m}$ & $17.9 \mathrm{~s}$ & 0 \\
\hline Q & Wingrock & Terrain & fails & - & - & $921 \mathrm{~m}$ & $9.1 \mathrm{~s}$ & 1 \\
\hline \hline Average & - & - & - & - & - & $1623 \mathrm{~m}$ & $14.6 \mathrm{~s}$ & - \\
\hline
\end{tabular}

Table 2: Evaluation of the aerial object tracking framework with the attitude estimation filter. For each scenario we show the remaining distance once the incoming traffic aircraft is successfully tracked, the remaining time to collision (TTC) and the total number of false tracks during the scenario. Note that for the evaluation without attitude estimation filter the traffic aircraft is detected as well, but the tracking framework fails to create a consistent track.

\subsection{Scenario Evaluation}

We evaluate the extended aerial object tracking framework on the existing Scenarios $A-M$ and four new wingrock Scenarios $N-Q$. Those scenarios are based on a standard head-on encounter while the own-ship continuously banks from left to right and reverse to induce heavy egomotion. In Table 2 the results are summarized and compared to the aerial object tracking framework without the attitude estimation extension. For each scenario the initial distance of the traffic aircraft with the corresponding remaining time to collision (TTC) is shown, once a valid track is available. The TTC is calculated based on the time once the track is declared valid and the time of the closest point of approach, assuming a constant closing speed. As a third performance measure we indicate the total number of false tracks during the complete scenario. For the Scenarios $A-M$ we achieve the same results as published in [Nussberger et al., 2015] which is a reasonable outcome because all of them are level flight. Therefore we do not repeat those results in Table 2.

Based on one of the new wingrock scenarios (Scenario $P$ ) we will now further analyze the dramatically improved tracking performance within high dynamic maneuvers. In Figure 10 the tracks from the tracking sphere are visualized as $2 \mathrm{D}$ surface plots. If the delayed attitude measurements are directly used, the tracker fails to create a consistent track and multiple individual tracks are initialized. Even worse, there are large gaps in between where the

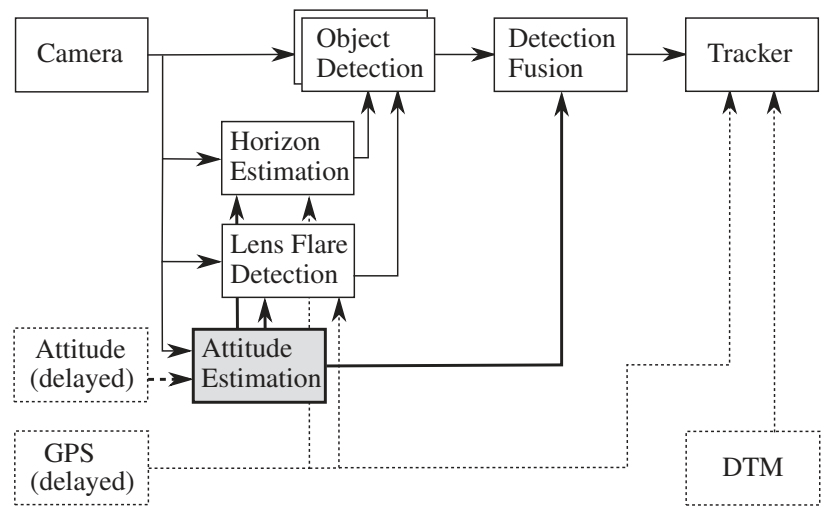

Figure 9: Aerial object tracking framework presented by [Nussberger et al., 2015], extended with the Attitude Estimation block to compensate for the delayed attitude measurements from the aircraft autopilot. The resulting offset from the delayed GPS position is not critical and can be neglected. tracking completely fails. This is a serious issue if the output of the optical DAA system shall be used further for an automatic avoidance maneuver. As soon as the attitude estimation filter is enabled as shown in the lower part of the figure, the tracker correctly maintains one single track for the approaching traffic aircraft - even though the own-ship continuously banks from left to right and reverse. Corresponding example frames for Scenario $N$ and $P$ with disabled and enabled attitude estimation filter are presented in Figure 12. For Scenario $O$ where the approaching traffic has to be detected in front of terrain we show a series of eight frames taken at a one second interval in Figure 11. As results show the track is declared valid at an initial distance of $\sim 1700 \mathrm{~m}$

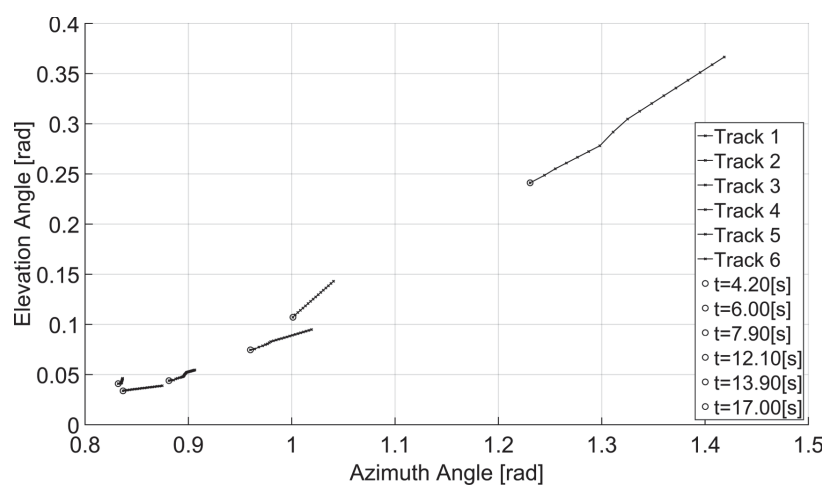

(a) Attitude Estimation disabled

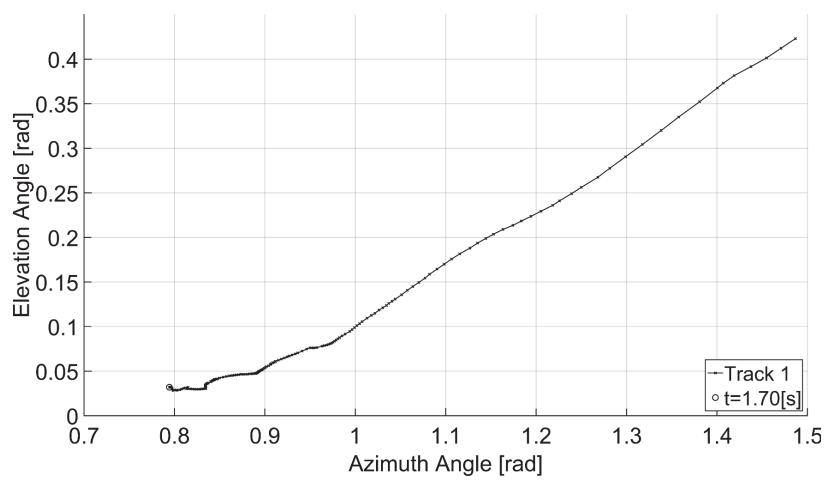

(b) Attitude Estimation enabled

Figure 10: Visualization of the tracks from the surface of the tracking sphere with disabled and enabled attitude filter for Scenario $P$. If the delayed attitude measurements are directly used, the detections are projected to wrong positions on the unit sphere and the tracker fails to create a consistent track. 


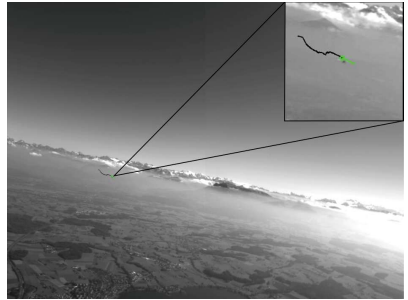

(a) $t_{a}=6 \mathrm{~s} / \mathrm{d}=1068 \mathrm{~m}$

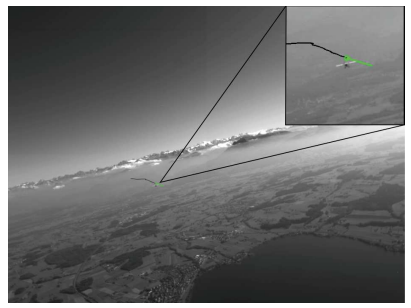

(e) $t_{a}=10 \mathrm{~s} / \mathrm{d}=623 \mathrm{~m}$

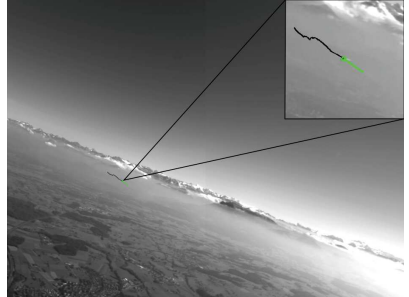

(b) $t_{a}=7 \mathrm{~s} / \mathrm{d}=956 \mathrm{~m}$

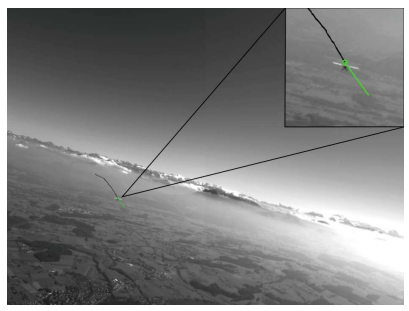

(f) $t_{a}=11 \mathrm{~s} / \mathrm{d}=511 \mathrm{~m}$

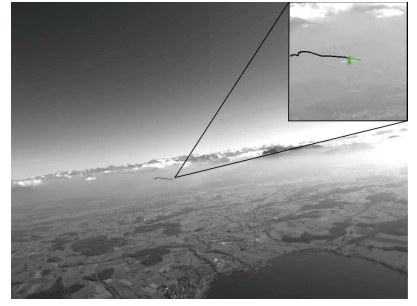

(c) $t_{a}=8 \mathrm{~s} / \mathrm{d}=846 \mathrm{~m}$

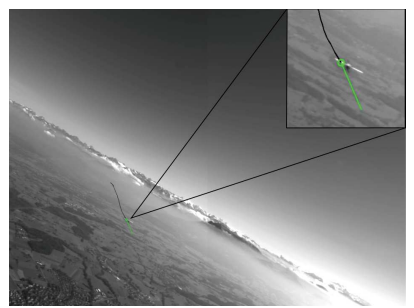

(g) $t_{a}=12 \mathrm{~s} / \mathrm{d}=399 \mathrm{~m}$

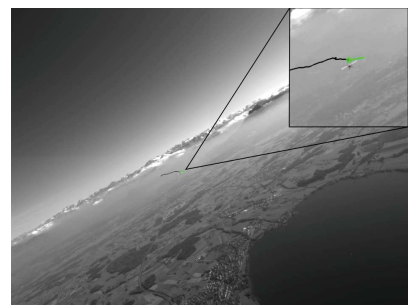

(d) $t_{a}=9 \mathrm{~s} / \mathrm{d}=735 \mathrm{~m}$

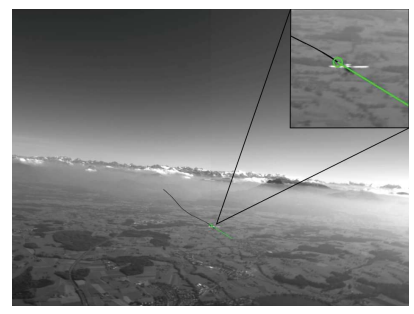

(h) $t_{a}=13 \mathrm{~s} / \mathrm{d}=290 \mathrm{~m}$

Figure 11: Example frames from the wingrock Scenario $O$ at different time steps. The track is shown with its one second prediction in green and the track history in black. The time $t_{a}$ indicates the total 'alive time' of the track. From frame (a) to (f) it can easily be seen that the period of the wingrock maneuver was approximately five seconds. This figure is best viewed in color.

and correctly maintained for the rest of the scenario. Detecting approaching traffic in front of terrain is generally more challenging compared to an object within the sky part of the image. One of the reasons is the typically small contrast between the traffic and the terrain.

The results achieved for the second scenario with terrain as background (Scenario $Q$ ) warrant a separate discussion because the initial track distance and the remaining TTC are significantly lower compared to the other scenarios. This is basically a result of the extreme environmental conditions: the sun is located in front but slightly above the camera resulting in heavy lens flare. The horizon is hazy and directly reflecting the sunlight which results in an exceptional dynamic range (note that this scenario was recorded with a bit depth of 8-bit) and the traffic aircraft initially approaches in front of a dark forest. As a result of this the traffic is first detected shortly before it is at $1000 \mathrm{~m}$. Once detections are available

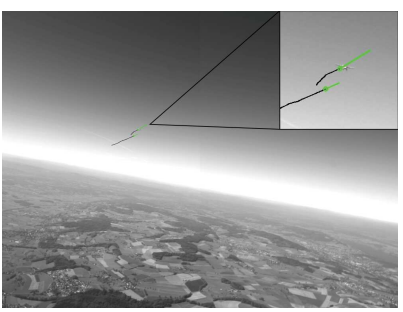

(a) Scenario $N$ : AEF off

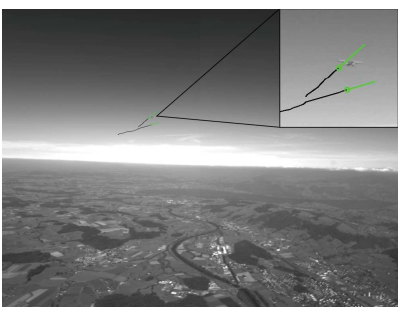

(c) Scenario $P$ : AEF off

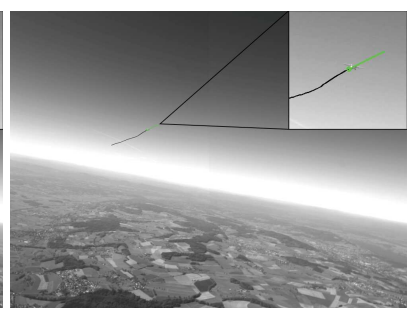

(b) Scenario $N$ : AEF on

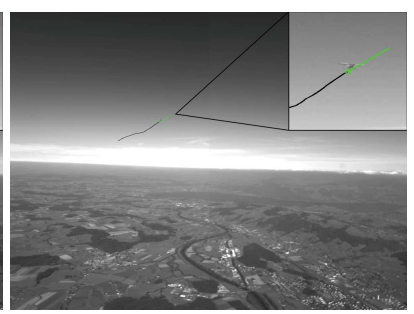

(d) Scenario P: AEF on
Figure 12: Example frames from Scenario $N$ and $P$ with disabled (AEF off) and enabled (AEF on) attitude estimation filter. Multiple tracks are created if the AEF is disabled because new detections are mapped to wrong positions on the tracking sphere (best viewed in color). the tracker is able to successfully track the object although lens flares repeatedly pass through the track due to the continuously changing roll angle. An example frame of the tracker output is shown in Figure 13.

\section{CONCLUSIONS}

We presented an efficient solution to calculate the angular rates of an aircraft from a multi camera rig based on the assumption of a pure rotation in between consecutive frames. By analyzing the influencing parameters we have shown that the pure rotation assumption is fulfilled as soon as the aircraft is operated at least $500 \mathrm{~m}$ above ground. Typical operation altitudes are usually way beyond $500 \mathrm{~m}$. With an indirect Kalman filter the angular rates are fused with delayed absolute attitude measurements available from a third-party RPAS autopilot. This allows estimating accurate absolute attitude angles corresponding to the current camera frames. We integrated the proposed attitude estimation filter

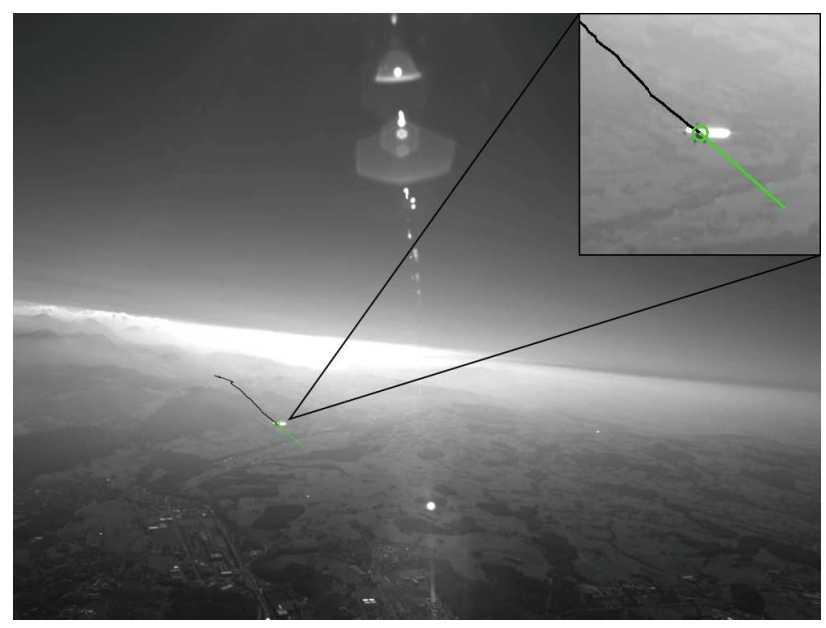

Figure 13: Example frame from Scenario $Q$ with enabled attitude estimation filter where the tracker correctly maintains one single track for the approaching traffic. As a result of the wingrock maneuver the lens flares continuously move across the image (best viewed in color). 
into an existing aerial object tracking framework and show that with this extension of the pipeline aerial objects are not only correctly tracked during level flight but also within high dynamic flight maneuvers. This is a key component for any EO DAA system because the maximum range where aerial objects can be detected might be smaller compared to other technologies. Therefore an automatic avoidance has to rely on consistent tracks, especially if an aggressive avoidance maneuver has to be executed due to nearby traffic. With the presented approach the aerial object tracking framework can be integrated into a third-party RPAS without the necessity of installing additional redundant sensors and the requirements on sensor data quality can be relaxed.

\section{ACKNOWLEDGMENTS}

This work is supported by armasuisse Science and Technology, affiliated with the Swiss Federal Department of Defense, Civil Protection and Sport.

\section{REFERENCES}

ASTM International, 2007. Standard specification for design and performance of an airborne sense-and-avoid system.

Carnie, R., Walker, R. and Corke, P., 2006. Image processing algorithms for UAV "sense and avoid". In: Proc. of International Conference on Robotics and Automation.

Challis, J. H., 1995. A procedure for determining rigid body transformation parameters. Journal of Biomechanics.

Davison, A. J., 2003. Real-time simultaneous localisation and mapping with a single camera. In: Proc of International Conference on Computer Vision.

Dey, D., Geyer, C., Singh, S. and Digioia, M., 2011. A cascaded method to detect aircraft in video imagery. International Journal of Robotics Research 30, pp. 1527-1540.

Diebel, J., 2006. Representing attitude: Euler angles, unit quaternions, and rotation vectors. Technical report, Stanford University.

Engel, J., Schöps, T. and Cremers, D., 2014. LSD-SLAM: Largescale direct monocular SLAM. In: Proc. of European Conference on Computer Vision.

European RPAS Steering Group, 2013. Roadmap for the integration of civil remotely-piloted aircraft systems into the european aviation system.

Federal Aviation Administration, 2013a. FAA order JO 7610.4 special operations.

Federal Aviation Administration, 2013b. Integration of civil unmanned aircraft systems (UAS) in the national airspace system (NAS) roadmap.
Forlenza, L., Fasano, G., Accardo, D. and Moccia, A., 2012. Flight performance analysis of an image processing algorithm for integrated sense-and-avoid systems. International Journal of Aerospace Engineering.

Forster, C., Pizzoli, M. and Scaramuzza, D., 2014. SVO: Fast semi-direct monocular visual odometry. In: Proc. of International Conference on Robotics and Automation.

International Civil Aviation Organization, 2011. ICAO cir 328, unmanned aircraft systems (UAS).

Kelly, J. and Sukhatme, G. S., 2010. A general framework for temporal calibration of multiple proprioceptive and exteroceptive sensors. In: Proc. of International Symposium on Experimental Robotics.

Klein, G. and Murray, D., 2007. Parallel tracking and mapping for small ar workspaces. In: Proc. of International Symposium on Mixed and Augmented Reality.

Kneip, L., Chli, M. and Siegwart, R., 2011. Robust real-time visual odometry with a single camera and an IMU. In: Proc. of British Machine Vision Conference.

Korn, B. and Edinger, C., 2008. UAS in civil airspace: Demonstrating "sense and avoid" capabilities in flight trials. In: Proc. of Digital Avionics Systems Conference.

Mejias, L., Lai, J. S. and Ford, J. J., 2012. Flight trial of an electro-optical sense-and-avoid system. In: Proc. of International Congress of the Aeronautical Sciences.

Nistér, D., Naroditsky, O. and Bergen, J., 2004. Visual odometry. In: Proc. of Conference on Computer Vision and Pattern Recognition.

Nussberger, A., Grabner, H. and Van Gool, L., 2014. Aerial object tracking from an airborne platform. In: Proc. of International Conference on Unmanned Aircraft Systems.

Nussberger, A., Grabner, H. and Van Gool, L., 2015. Robust aerial object tracking in images with lens flare. In: Proc. of International Conference on Robotics and Automation.

Owen, M., Duffy, S. and Edwards, M., 2014. Unmanned aircraft sense and avoid radar: Surrogate flight testing performance evaluation. In: Proc. of Radar Conference.

Tasoulis, D. K., Adams, N. M. and Hand, D. J., 2007. Delayed measurements and the Kalman filter. Technical report, Imperial College London, Dept. of Math.

Trawny, N. and Roumeliotis, S. I., 2005. Indirect Kalman filter for 3D attitude estimation. Technical report, University of Minnesota, Dept. of Comp. Sci. \& Eng.

Utt, J., McCalmont, J. and Deschenes, M., 2004. Test and integration of a detect and avoid system. In: Proc. of AIAA "Unmanned Unlimited" Technical Conference.

Wolfe, R. C., 2003. NASA ERAST non-cooperative DSA flight test. Technical report, National Aeronautics and Space Administration. 